\title{
L'approche qualitative et la recherche en éducation
}

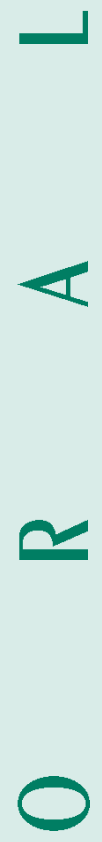

\section{L}

es méthodes de recherche utilisées en médecine proviennent principalement des sciences phy elles sappui ent sur l'échantillonnage au hasard, mesure et les analyses stati stiques. L'essai clinique en constitue l'étalon d'or comme en témoignent les pro cessus d'élaboration des pratiques cl iniques basées sur les évidences. Cependant, en matière d'éducation, on ne peut tout aborder par les méthodes quantitatives. On n'a qu'à penser à toutes les questions concernant l'acquisition des attitudes et de l'éthique, le rôle des modèles de rôle ou les difficultés d'apprenti ssage ren contrées au cours de la formation des médecins. La recherche qualitative offre des moyens d'étudier ces phénomènes et ainsi, d'enrichir le domaine de la pédagogi e des sciences de la santé.

Processus de recherche basé sur des méthodes spéci fiques et reconnues, la recherche qualitative vise la compréhension de phénomènes complexes, humains ou sociaux, qui ne peuvent facilement être réduits en éléments chiffrables ou être décontextualisés. Le pro cessus a lieu dans un milieu naturel plutôt qu'en milieu contrôlé comme dans un laboratoire. D e plus, le chercheur simplique lui même dans la collecte et I'analyse de données en pri orisant le sens que les par ticipants donnent au phénomène étudié. Enfin, le chercheur décrit le processus de recherche et les résul tats dans une narration au style expressif et persuasif (Creswell, 1998). Les principales méthodes qualita tives sont l'étude de cas, la phénoménologie, l'ethno graphie et l'approche de la théorie ancrée. En péda gogie on retrouve aussi la méthode de la science action, basée sur les concepts de l'apprentis sage expérientiel. Un chercheur choisira une de ces méthodes lorsque (1) la question de recherche a trait à la nature d'un phénomène ou aux processus qui y sont reliés, plutôt qu'aux liens de causalité ; (2) il y a un phénomène dont les concepts et les variables sont peu définis ou qu'il est nécessaire de développer une théorie ; (3) on recherche une description \& une com préhension approfondie d'un phénomène ou d'un processus; (4) on doit étudier le phénomène dans son contexte naturel afin d'en augmenter la crédibilité et la transférabilité ; (5) le chercheur désire apprendre au sujet d'un phénomène en sy impliquant luimême.

Ces méthodes ne sont pas des substituts faciles aux méthodes quantitatives. Bien au contraire! Elles exi gent beaucoup de rigueur tant sur les plans de la collecte que de l'analyse et de l'interprétation des données. Q uiconque a effectué des entrevues semistructurées au sujet d'un phénomène et qui en a ensuite analysé le verbatim pour en tirer une recons truction du phénomène riche, élaborée, globale en sait quelque chose! Ainsi, les études qualitati ves solides répondent à des critères de rigueur tout aussi sévères, mais sdifférents, que ceux des études quantita tives, y comprisles essais cliniques, La principale dif férence avec les études quantitatives se situe au plan de la généralisation parce que les données ne sont pas recueillies auprès d'échantillons au hasard mais plu tôt auprès d'échantillons de convenance. Les cher cheurs qualitatifs recherchent, en effet, le maximum de variation du phénomène étudié et choisissent les sujets en fonction de cet objectif. La transférabilité, plutôt que la généralisation, repose alors sur la des cription détaillée de l'échantillon permettant ainsi aux lecteurs de comparer leurs populations à celle du chercheur. D e plus, la triangulation entre les données dans une même étude (entrevues, documents, focusgroup) et la triangulation des résultats avec d'autres études ayant utilisé d'autres méthodes contribuent à la transférabilité. Enfin, le double codage et la vali dation par les partici pants assurent la validité, qu'on dési gne plutôt par le terme crédibilité en recherche qualitative.

Ainsi, plutôt que d'un débat concurrentiel entre les méthodes quantitatives et qualitatives, il est certain que le domaine de la pédagogie des sci ences de la santé bénéfi cie davantage d'une connai ssance de ce que chaque approche et chaque méthode est suscep tible de contribuer. C'est la nature de la question de recherche qui guide le chercheur dansle choix métho dologique d'une étude. Plus un chercheur est ouvert et souple aux deux approches et à plusi eurs méthodes de recherche, plus il possède d'outils pour répondre à ses questions.

L'article présenté dans ce numéro par Luc Côté et Jean Turgeon permet d'aller plus loin dans ce thème en commentant comment fai reune lecture critique et productive des articles décrivant des travaux de recherche effectués avec une méthode quali tative.

Johanne GOUDREAU

U niversité de M ontréal \& U nivers té du Q uébec à H ull johannegoudreau@ssss.gouv.qc.ca 